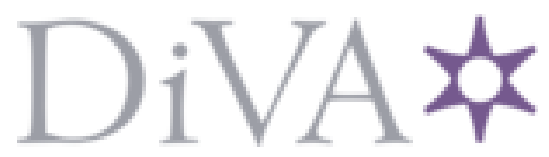

http://www.diva-portal.org

This is the published version of a paper published in Physical Review E. Statistical, Nonlinear, and Soft Matter Physics.

Citation for the original published paper (version of record):

Lundow, P-H., Campbell, I A. (2016)

Non-self-averaging in Ising spin glasses and hyperuniversality.

Physical Review E. Statistical, Nonlinear, and Soft Matter Physics, 93(1): 012118

http://dx.doi.org/10.1103/PhysRevE.93.012118

Access to the published version may require subscription.

N.B. When citing this work, cite the original published paper.

Permanent link to this version:

http://urn.kb.se/resolve?urn=urn:nbn:se:umu:diva-1 16080 


\title{
Non-self-averaging in Ising spin glasses and hyperuniversality
}

\author{
P. H. Lundow \\ Department of Mathematics and Mathematical Statistics, Umeå University, SE-901 87, Sweden \\ I. A. Campbell \\ Laboratoire Charles Coulomb (L2C), UMR 5221 CNRS-Université de Montpellier, Montpellier, France
}

(Received 28 August 2015; published 11 January 2016)

\begin{abstract}
Ising spin glasses with bimodal and Gaussian near-neighbor interaction distributions are studied through numerical simulations. The non-self-averaging (normalized intersample variance) parameter $U_{22}(T, L)$ for the spin glass susceptibility [and for higher moments $U_{n n}(T, L)$ ] is reported for dimensions 2,3,4,5, and 7. In each dimension $d$ the non-self-averaging parameters in the paramagnetic regime vary with the sample size $L$ and the correlation length $\xi(T, L)$ as $U_{n n}(\beta, L)=\left[K_{d} \xi(T, L) / L\right]^{d}$ and so follow a renormalization group law due to Aharony and Harris [Phys. Rev. Lett. 77, 3700 (1996)]. Empirically, it is found that the $K_{d}$ values are independent of $d$ to within the statistics. The maximum values $\left[U_{n n}(T, L)\right]_{\max }$ are almost independent of $L$ in each dimension, and remarkably the estimated thermodynamic limit critical $\left[U_{n n}(T, L)\right]_{\max }$ peak values are also practically dimension-independent to within the statistics and so are "hyperuniversal." These results show that the form of the spin-spin correlation function distribution at criticality in the large $L$ limit is independent of dimension within the ISG family. Inspection of published non-self-averaging data for three-dimensional Heisenberg and $X Y$ spin glasses the light of the Ising spin glass non-self-averaging results show behavior which appears to be compatible with that expected on a chiral-driven ordering interpretation but incompatible with a spin-driven ordering scenario.
\end{abstract}

DOI: 10.1103/PhysRevE.93.012118

\section{INTRODUCTION}

The non-self-averaging parameter, usually noted $A$ or $U_{22}$, represents the normalized intersample variability for systems such as diluted ferromagnets or spin glasses where the microscopic structures of the interactions within individual samples are not identical. The parameter is defined for ferromagnets as the intersample variance of the susceptibility normalized by the mean susceptibility squared [1],

$$
U_{22}(\beta, L)=\left[\frac{\sigma_{\chi}(T, L)}{\chi(T, L)}\right]^{2}=\frac{\operatorname{var}\left(\left\langle q^{2}\right\rangle\right)}{\left[\left\langle q^{2}\right\rangle\right]^{2}},
$$

where $\sigma(T, L)$ is the standard deviation of the equilibrium sample-by-sample distribution of the susceptibility. We denote by $\langle\cdots\rangle$ the thermal mean for a single sample and by $[\cdots]$ the sample mean. In Ising spin glasses (ISGs) the spin glass susceptibility replaces $\chi$. The non-self-averaging definition can be widened to other observables [1]; we will also discuss the behavior of non-self-averaging of higher moments $\left\langle q^{3}\right\rangle$ and $\left\langle q^{4}\right\rangle$ of the spin overlap parameter $q$ defined in Eq. (5). In general the $U_{n n}$ parameter is defined as

$$
U_{n n}(\beta, L)=\frac{\operatorname{var}\left(\left\langle|q|^{n}\right\rangle\right)}{\left[\left\langle|q|^{n}\right\rangle\right]^{2}} .
$$

Aharony and Harris [1] gave a fundamental renormalization group discussion of non-self-averaging in diluted ferromagnets, which can be applied also to spin glass models. First, they showed that in the paramagnetic regime, at temperatures above the critical temperature, $U_{22}$ (which they referred to as $R_{\chi}$ ) behaves as

$$
U_{22}(T, L) \sim(\xi(T, L) / L)^{d},
$$

where $d$ is the dimension of the system and $\xi(T, L)$ is the standard finite size second moment correlation length (see, for instance, Ref. [2]). This rule can be understood in a simple physical picture: the intersample variability depends on the ratio of the sample volume to the correlated volume. Roughly, each sample is contained in a "box" of volume $L^{d}$. When this box volume is much larger than the correlated volume $\xi(T)^{d}$, all samples will have essentially identical properties; when the inverse is true, each sample has its own individual properties.

Then at the critical point $T_{c}$ where $\xi(T)$ diverges in the thermodynamic limit (ThL), $U_{22}\left(T_{c}, L\right)$ becomes independent of $L$ even when $L$ tends to infinity [1]. In this strongly non-self-averaging regime the observables for each individual sample have different properties. The passage as a function of temperature in the thermodynamic limit from "all samples identical" (randomness irrelevant) to "all samples different" (randomness relevant) is a fundamental signature of the physical meaning of ordering in systems with disorder or in spin-glass-like systems. Aharony and Harris show that the value of $U_{22}\left(T_{c}, L\right)$ in the limit of large $L$ should be universal for ferromagnets with different forms of disorder in a given dimension. We find empirically that within the ISG family this critical parameter is practically dimension-independent, i.e., "hyperuniversal."

We report non-self-averaging measurements in near neighbor interaction ISGs having dimensions 2, 3, 4, 5, and 7, with bimodal or Gaussian near neighbor interaction distributions. There is considerable regularity in behavior throughout all this range of $d$, which includes the special cases $d=2$ where $T_{c}=0$ and $d=7$, which is above the upper critical dimension $d=6$. In the paramagnetic regime $U_{22}(T, L)=$ $\left[K_{d} \xi(T, L) / L\right]^{d}$ with $K_{d} \approx 2.5$ for all $d$ studied, to within statistical accuracy. Second, the peak in $U_{22}(T, L)$ as a function of $T$ for fixed $L$ has a value $U_{22}(\max )$ for each $L$ which, after weak small size effects, is independent of $L$ to within the statistics and almost independent of $d, U_{22}(\max ) \approx 0.205$. 
The location of the peak $T\left(U_{22}(\max )\right)$ approaches $T_{c}$ from the paramagnetic regime (higher $T$ ) for $d<4$ and from the ordered regime (lower $T$ ) for $d>4$. The same rules are followed for the higher moments of the spin-spin correlations.

\section{SIMULATIONS}

The standard ISG Hamiltonian is

$$
\mathcal{H}=-\sum_{i j} J_{i j} S_{i} S_{j}
$$

with the near-neighbor symmetric distributions normalized to $\left\langle J_{i j}^{2}\right\rangle=1$. The normalized inverse temperature is $\beta=$ $\left(\left\langle J_{i j}^{2}\right\rangle / T^{2}\right)^{1 / 2}$. The Ising spins live on simple hypercubic lattices with periodic boundary conditions. The spin overlap parameter is defined as usual by

$$
q=\frac{1}{L^{d}} \sum_{i} S_{i}^{A} S_{i}^{B},
$$

where the sum is taken over all spins and $A$ and $B$ indicate two copies of the same system. The spin glass susceptibility is then defined as usual: $\chi(\beta, L)=L^{d}\left[\left\langle q^{2}\right\rangle\right]$.

The equilibration techniques (which are different in dimension 2) are described in Refs. [3,4]. On a technical level, it turns out that the values of $U_{22}$ and particularly the peak values can fluctuate slightly in an irregular manner at each size, possibly because of outliers in some of the distributions. Also, the values depend sensitively on strict equilibration having been achieved.

\section{DIMENSION 2}

It is well established that short range ISGs in dimension 2 only order at $T=0[5,6]$. The Gaussian ISG has a nondegenerate ground state and a continuous energy level distribution. The bimodal ISG has an effectively continuous energy level regime down to an $L$-dependent crossover temperature $T^{*}(L)$ below which the thermodynamics are dominated by the massively degenerate ground state [7]. This is a finite-size regime; in the ThL regime the bimodal ISG can be considered to have an effectively continuous energy level distribution similar to that of the Gaussian ISG.

Measurements on two bimodal models and the Gaussian model ISG in dimension 2 [8] show a clear scaling of $U_{22}(T, L)$ as a function of $\xi(T, L) / L$, with all the maxima in $U_{22}(T, L)$ close to 0.20 . We show for the standard bimodal ISG in dimension 2 (see Fig. 1) the data scaled against $\xi(T, L) / L$ on a $\log$-log plot. This brings out the fact (not mentioned in Ref. [8]) that for temperatures above the peak location temperature, the Aharony-Harris rule [1] $U_{22}(T, L)=\left[K_{2} \xi(T, L) / L\right]^{2}$ holds, with $K_{2}=2.5(1)$. Below the peak obvious finite size effects due to the crossover to the ground state-dominated regime set in.

From the same simulation runs, data for the higher moments $\left\langle q^{3}\right\rangle(T, L)$ and $\left\langle q^{4}\right\rangle(T, L)$ were obtained, and the values of the normalized variances $U_{33}(T, L)$ and $U_{44}(T, L)$ were evaluated. Equivalent plots to Fig. 1 are shown for $U_{33}(T, L)$ and $U_{44}(T, L)$ in Figs. 2 and 3 with $U_{33}(T, L)=[3.29 \xi(T, L) / L]^{2}$ and $U_{44}(T, L)=[4.36 \xi(T, L) / L]^{2}$.

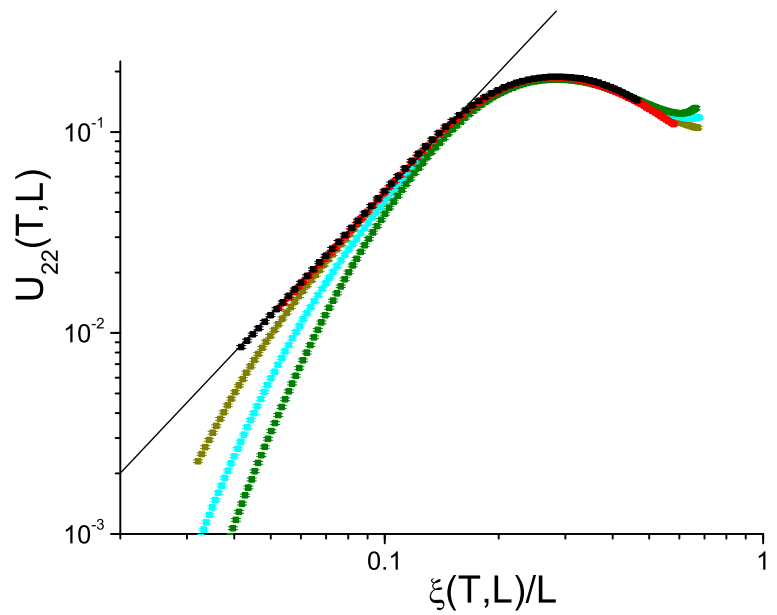

FIG. 1. Bimodal 2D ISG. Non-self-averaging parameter $U_{22}(T, L)$ against the normalized correlation length $\xi(T, L) / L . L=$ $12,16,24,32,48,64,96,128$ from right to left. The straight line has slope 2 .

The same data are presented as $U_{22}(T, L)$ against $T$ for fixed $L$ in Fig. 4; the peak location is moving towards $T=0$ with increasing $L$, and the maximum value is very gradually growing with increasing $L$. A simple extrapolation of the peak data from $L=4$ to $L=128$ indicates a limiting infinite $L$ peak value close to 0.200 .

The $U_{33}(T, L)$ and $U_{44}(T, L)$ peak values and positions evolve in a very similar way to the $U_{22}(T, L)$ peaks, extrapolating to large $L$ limit values $U_{33}=0.38$ and $U_{44}=$ 0.60 (Figs. 5 and 6). On the low-temperature side of the bimodal data, a minimum in each of the $U_{n n}(T, L)$ at an $L$-dependent temperature followed by a plateau (see Ref. [8]) provides a clear indication of the crossover from the effectively continuous energy level regime to the degenerate ground state-dominated regime. For the largest sizes, this crossover lies below the lowest temperatures at which measurements were carried out.

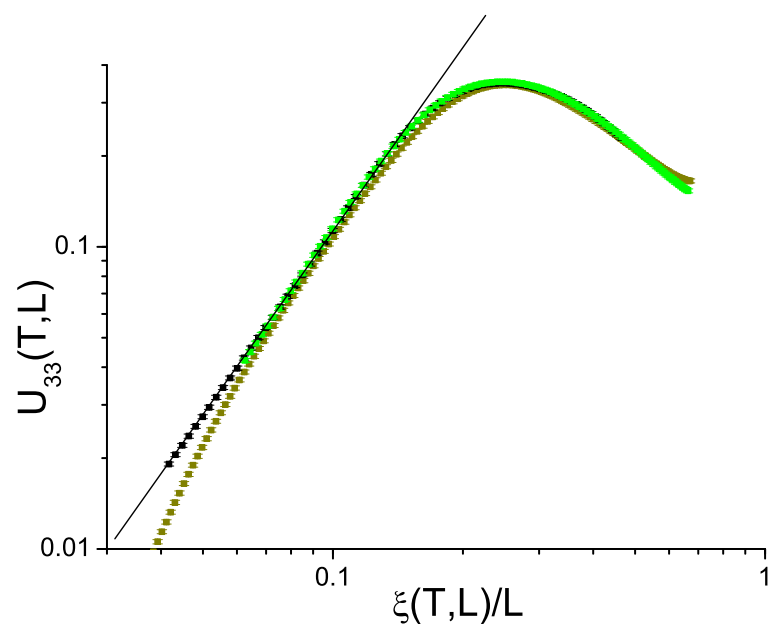

FIG. 2. Bimodal 2D ISG. Non-self-averaging parameter $U_{33}(T, L)$ against the normalized correlation length $\xi(T, L) / L$. $L=24,32,48,64,96,128$ from right to left. The straight line has slope 2 . 


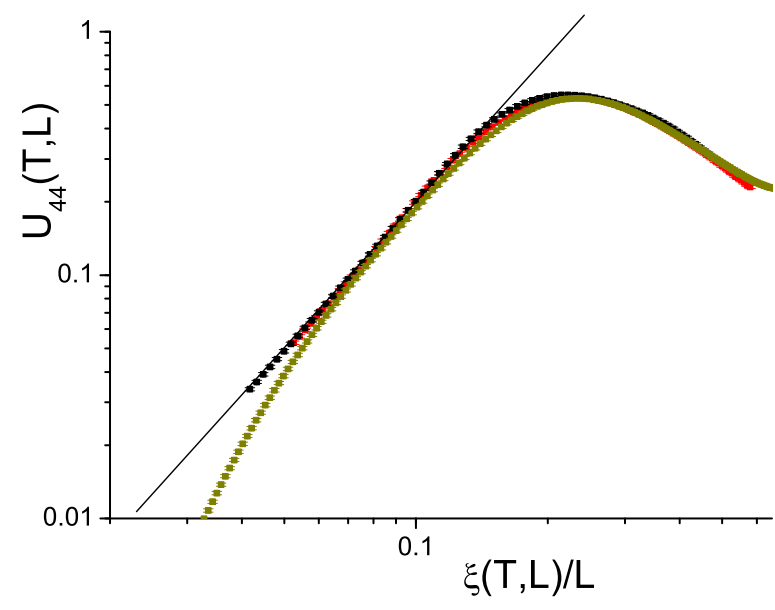

FIG. 3. Bimodal 2D ISG. Non-self-averaging parameter $U_{44}(T, L)$ against the normalized correlation length $\xi(T, L) / L$. $L=24,48,64,128$ from right to left. The straight line has slope 2 .

Data for $U_{22}(T, L)$ for the Gaussian ISG (not shown) are very similar to the bimodal data, except that there is of course no crossover effect.

For both the bimodal and the Gaussian two-dimensional (2D) models (Fig. 7) the peak position scales in the standard manner as

$$
\left[T_{\max }(L)-T_{c}\right]=A L^{-1 / v}\left[1+a L^{-\omega}\right],
$$

where $T_{c}=0$ and $v$ is the correlation length exponent, which is already accurately estimated (see Ref. [5] for the Gaussian and Ref. [4] for the bimodal) and a correction term is needed. Thus we have $U_{22}$ fits: $T_{\max }(L)=1.2 L^{-1 / 3.5}\left[1+0.71 L^{-0.45}\right]$ for the Gaussian and $T_{\max }(L)=1.12 L^{-1 / 4.8}\left[1+2.54 L^{-0.45}\right]$ for the bimodal. The $T_{\max }(L)$ values for $U_{33}$ and $U_{44}$ are not identical to the $U_{22}$ values, but the scaling fits are of very similar form.

[In a rather different context (see Fig. 2[a] of Ref. [9]) the non-self-averaging parameter peak position for the threedimensional (3D) random field Ising model moment at zero

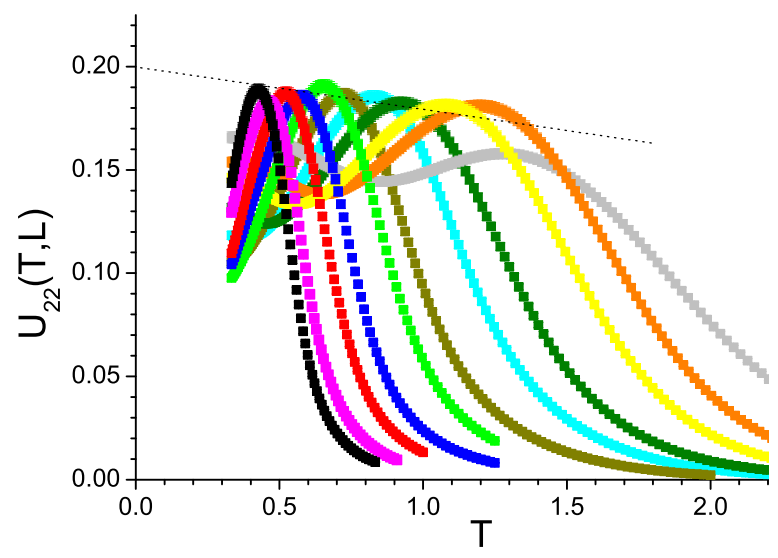

FIG. 4. Bimodal 2D ISG. Non-self-averaging parameter $U_{22}(T, L)$ against the temperature $T . L=128,96,64,48,32,24,16$, $12,8,6,4$ from left to right. The straight line indicates extrapolation to criticality at $T=0$.



FIG. 5. Bimodal 2D ISG. Non-self-averaging parameter $U_{33}(T, L)$ against the temperature $T . L=128,96,64,48,32,24$, $16,12,8,6$ from left to right. The straight line indicates extrapolation to criticality at $T=0$.

temperature has been shown to behave as a function of $L$ in a remarkably similar way, with fields $h$ replacing temperatures and no correction term. The observed peak heights are independent of $L$.]

The zero temperature infinite size limit can be defined in two ways. Taking the successive limits $L \rightarrow \infty, T \rightarrow 0$ gives an extrapolated value $U_{22}(0, \infty)=0.190(5)$ for both bimodal and Gaussian models, while the successive limits $T \rightarrow 0, L \rightarrow \infty$ gives a value $\approx 0$ in the Gaussian case; with the present data it is hard to estimate in the bimodal model because of the crossover.

\section{DIMENSION 3}

The bimodal ISG in dimension 3 has a transition temperature for which the most recent estimate is $T_{c}=1.102(3)$ $[2,10,11]$, and the Gaussian ISG has a transition temperature estimated to be $T_{c}=0.951(9)$ [2]. The critical values of the dimensionless correlation length ratio $[\xi(T, L) / L]_{c}$ are estimated to be $0.652(3)$ and $0.635(10)$, respectively.

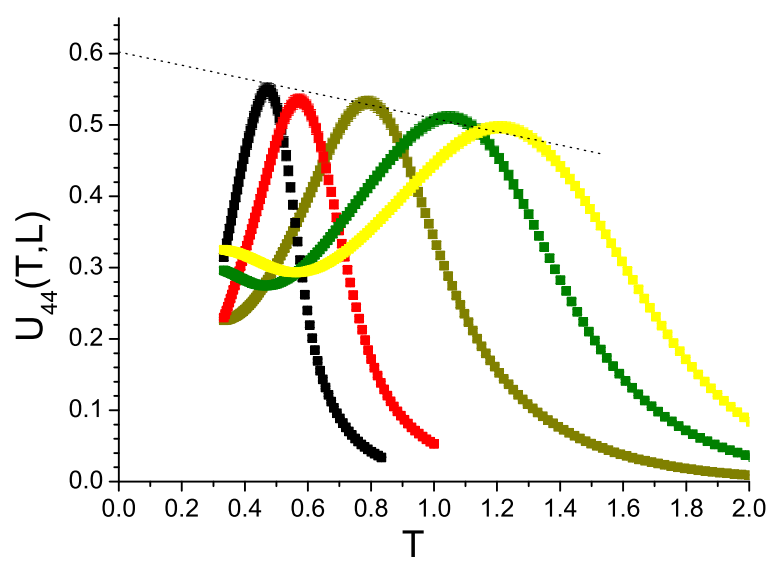

FIG. 6. Bimodal 2D ISG. Non-self-averaging parameter $U_{44}(T, L)$ against the temperature $T . L=8,16,32,64,128$ from right to left. The straight line indicates extrapolation to criticality at $T=0$. 


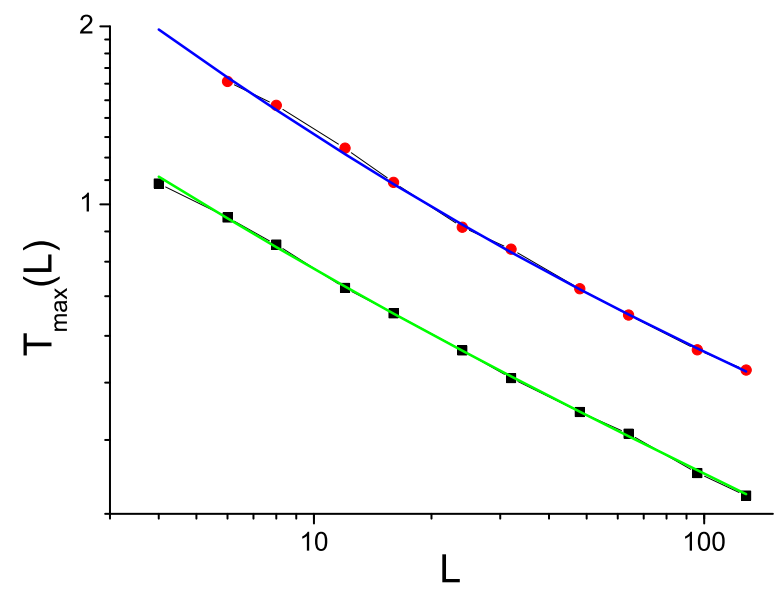

FIG. 7. Temperature of the maximum of the non-self-averaging parameter $U_{22}(T, L)$ against size $L$ for the 2D ISGs. Bimodal: data red circles, fit upper blue curve. Gaussian: data black squares, fit lower green curve. Fit expressions are in the text.

Hasenbusch et al. [10] have generously posted their raw tabulated simulation data for the bimodal ISG in dimension 3 as Supplemental Material with their publication. In addition to the present measurements on $2^{13}$ samples of sizes $L=4,6$, $8,10,12$ we have extracted a selection of values of $U_{22}(\beta, L)$ from the tables of Ref. [10], choosing the data sets with the largest numbers of temperatures, $L=16,20,24$. In each case the data correspond to measurements on about $10^{5}$ samples.

The $U_{22}(T, L)$ bimodal data in $3 \mathrm{D}$ have almost $L$ independent peak values $U_{22}(T, L)_{\max }=0.207(3)$ with peak locations tending gradually downwards towards $T_{c}$ as $L$ increases (Figs. 8 and 9; see Ref. [12], which also observed a very similar peak height for a $3 \mathrm{D}$ next-nearest-neighbor bimodal model). Small fluctuations as a function of $L$ can be put down to residual equilibration differences as the statistical errors in these data are very small because of the large numbers

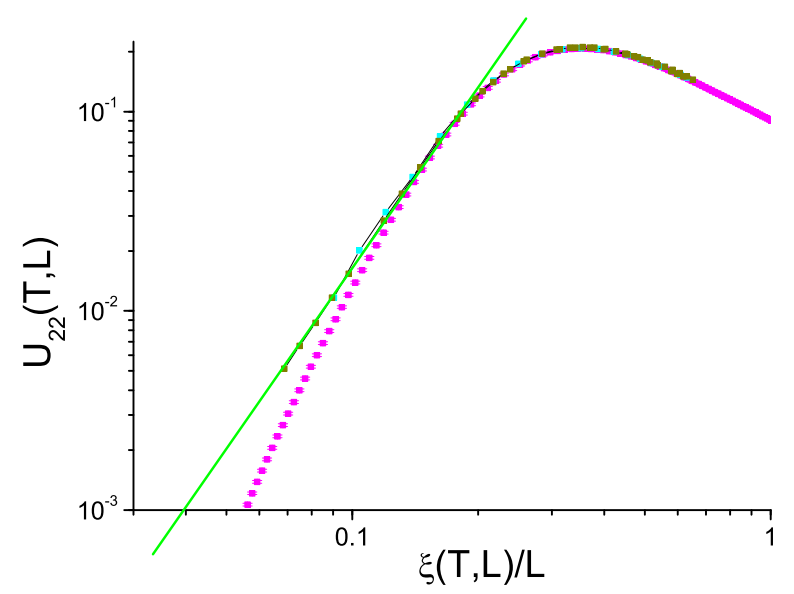

FIG. 8. Bimodal 3D ISG. Non-self-averaging parameter $U_{22}(T, L)$ against the normalized correlation length $\xi(T, L) / L$. $L=10,12,16,20,24$ (pink, purple, cyan, green, black) right to left. $L=16,20,24$ from Ref. [10]. The straight line has slope 3 .

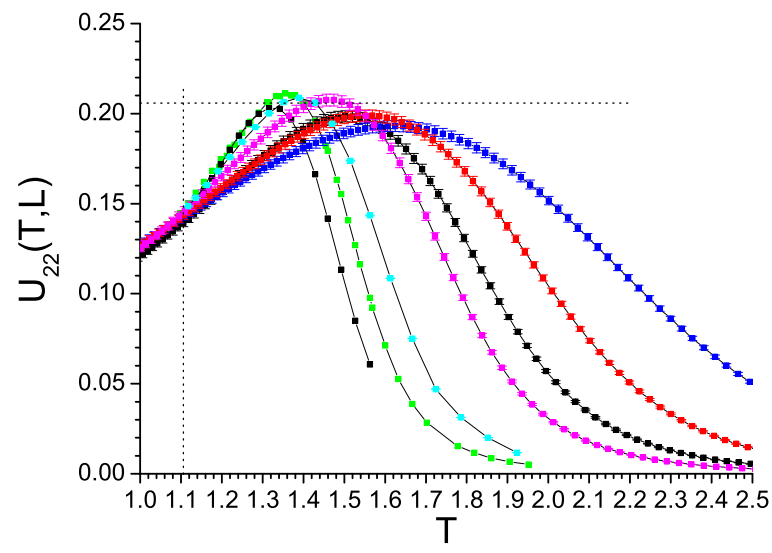

FIG. 9. Bimodal 3D ISG. Non-self-averaging parameter $U_{22}(T, L)$ against temperature $T . L=4,6,8,10,12,16,20,24$ (blue, red, black, pink, purple, cyan, green, brown) right to left. $L=16,20$, 24 from Ref. [10]. Vertical line $T_{c}$.

of samples. At the critical temperature the finite-size scaling limit for $\left[T \rightarrow T_{c}, L \rightarrow \infty\right]$ is $U_{22}\left(T_{c}, L\right)=0.147(3)$ [10].

When scaled against $\xi(T, L) / L$, in the paramagnetic range $U_{22}(T, L)=\left[K_{3} \xi(T, L) / L\right]^{3}$ following the Aharony-Harris law, with $K_{3}=2.6(1)$ (Fig. 8). The peak locations correspond to $\xi(T, L) / L \approx 0.35$. Alternatively the peak locations can be approximately scaled as $\left[T_{\max }-1.1\right]=1.07 L^{-1 / 2.1}$, or if the value of $v=2.56$ from Ref. [11] is imposed with a correction term, as $\left[T_{\max }-1.1\right]=0.8 L^{-1 / 2.56}\left[1+0.8 L^{-1}\right]$.

In the large $L$ limit, the $U_{33}$ and $U_{44}$ peak locations are tending to $T_{c}$, and the peak values extrapolate to $U_{33} \sim 0.39$ and $U_{44} \sim 0.61$ (Figs. 10 and 11).

\section{DIMENSION 4}

$U_{22}(T, L), U_{33}(T, L)$ and $U_{44}(T, L)$ data for the Gaussian ISG in dimension 4 are shown in Figs. 12, 13, 14, and 15. The data correspond to $N=8192$ samples for each $L$. The critical temperature is $T_{c}=1.80(3)$ [2,3], and the finite size critical value for the normalized correlation length ratio $[\xi / L]_{c}=$ $0.440(5)[2,3]$. Scaling against the normalized correlation length (Fig. 12), $U_{22}(T, L)=\left(K_{4} \xi(T, L) / L\right)^{4}$ again following

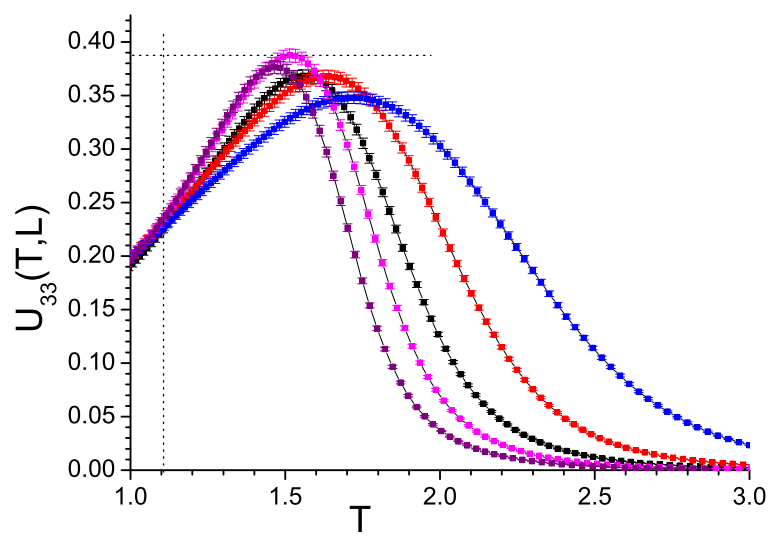

FIG. 10. Bimodal 3D ISG. Non-self-averaging parameter $U_{33}(T, L)$ against temperature $T . L=4,6,8,10,12$ (blue, red, black, pink, purple) right to left. Vertical line $T_{c}$. 


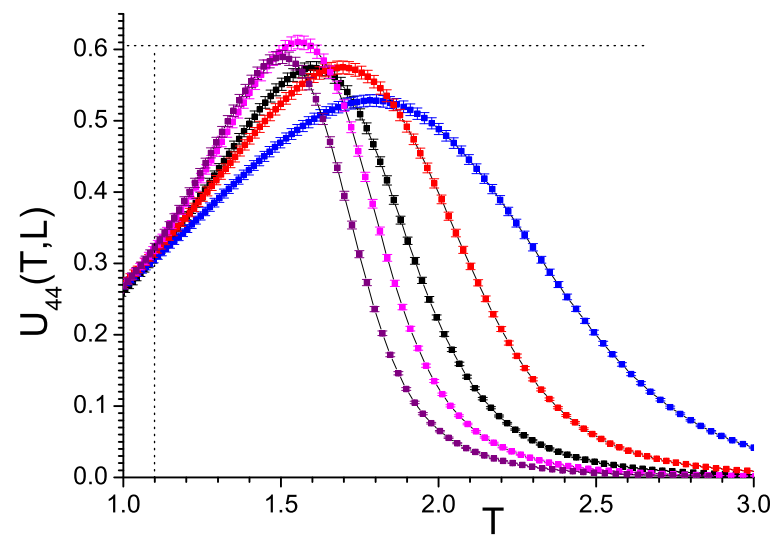

FIG. 11. Bimodal 3D ISG. Non-self-averaging parameter $U_{44}(T, L)$ against temperature $T . L=4,6,8,10,12$ (blue, red, black, pink, purple) right to left. Vertical line $T_{c}$.

the Aharony-Harris law, with $K_{4}=2.7(1)$ and peaks located at $\xi(T, L) / L=0.43(2)$ so very close to $\xi\left(T_{c}, L\right) / L$.

Data obtained for the four-dimensional (4D) bimodal ISG (not shown) follow a very similar pattern with the same peak height. The locations of the $U_{n n}(T, L)$ peaks are almost independent of $L$. This was noted for $U_{22}(T, L)$ in the 4D Gaussian ISG in Ref. [12] and in Ref. [13] for a bond-diluted bimodal model; it follows from the proximity of the peak $\xi(T, L) / L$ and critical $\xi\left(T_{c}, L\right) / L$ values. For the bond-diluted bimodal model, the peak height is again $\approx 0.205$ [13]. Because of the quasi- $L$-independence, the peak location extrapolated to infinite size provides an estimate for $T_{c}$, which is limited in precision only by the statistical uncertainties; no scaling of the peak position against $L$ is possible in this dimension.

The Gaussian $U_{n n}(T, L)$ peak heights become independent of $L$ to within the statistical errors after weak finite size effects for small $L$ (Figs. 13, 14, and 15). The stability of the $U_{n n}(T, L)$ peak heights as $L$ is varied turns out to be a useful empirical criterion for the quality of equilibration.



FIG. 12. Gaussian 4D ISG. Non-self-averaging parameter $U_{22}(T, L)$ against the normalized correlation length $\xi(T, L) / L . L=4$, $6,8,10,12$ (blue, red, black, pink, green), right to left. The straight line has slope 4 .

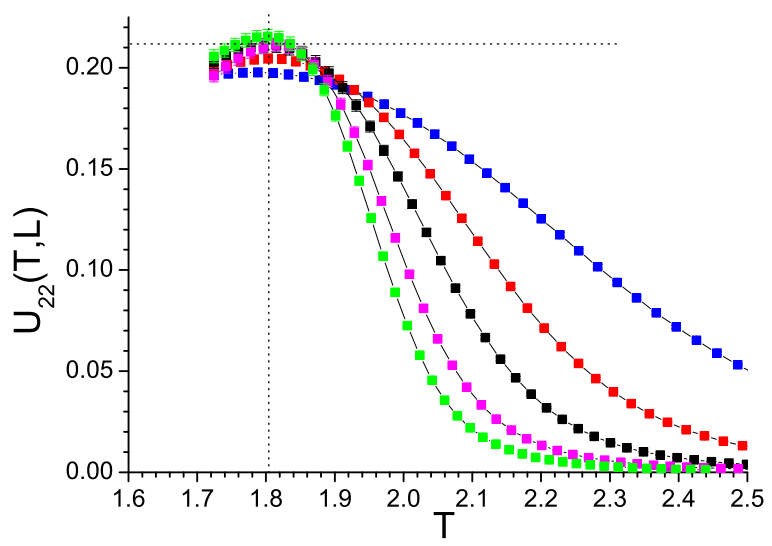

FIG. 13. Gaussian 4D ISG. Non-self-averaging parameter $U_{22}(T, L)$ against the temperature $T . L=4,6,8,10,12$ (blue, red, black, pink, green) from right to left. The horizontal line is an extrapolation to criticality at $T=T_{c}$ (vertical line).

Alternatively, considering the $U_{n n}(T, L)$ as dimensionless variables, the intersections of the curves for fixed $L$ should also give a criterion for estimating $T_{c}$, but the statistical fluctuations and corrections to scaling affect the intersections much more drastically than they do the peak location, which means that this is an imprecise criterion in the 4D case as noted in Ref. [13].

\section{DIMENSION 5}

$U_{22}(T, L), U_{33}(T, L)$, and $U_{44}(T, L)$ data for the Gaussian ISG in dimension 5 are shown in Figs. 16, 17, 18, and 19. The data correspond to 4096 samples for each $L$. The critical temperature is $T_{c}=2.390(5)$, and the finite size critical value for the normalized correlation length ratio $[\xi / L]_{c} \approx 0.45$ [14]. We are not aware of other comparable simulation measurements in this dimension. Data obtained for the 5D bimodal ISG (not shown) are very similar. The $U_{n n}(T, L)$ peak heights become independent of $L$ to within the statistical errors after weak finite size effects for small $L$.

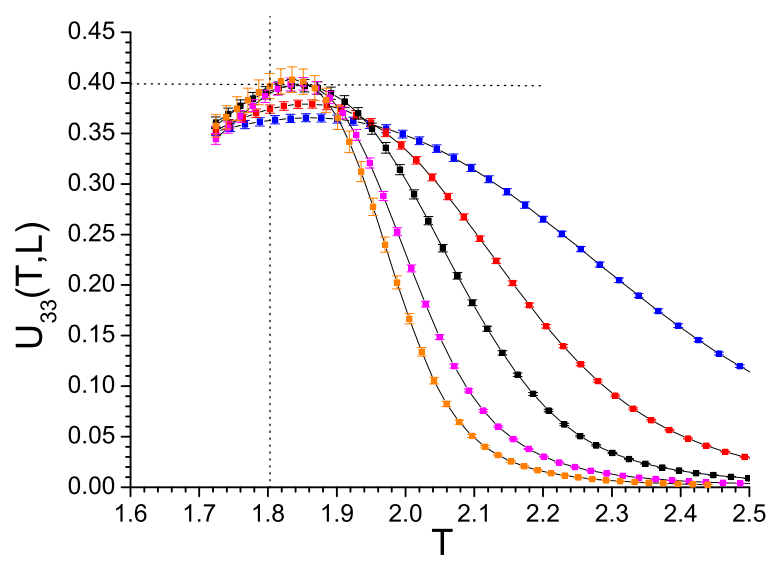

FIG. 14. Gaussian 4D ISG. Non-self-averaging parameter $U_{33}(T, L)$ against the temperature $T . L=4,6,8,10,12$ (blue, red, black, pink, orange) from right to left. The horizontal line is an extrapolation to criticality at $T=T_{c}$ (vertical line). 


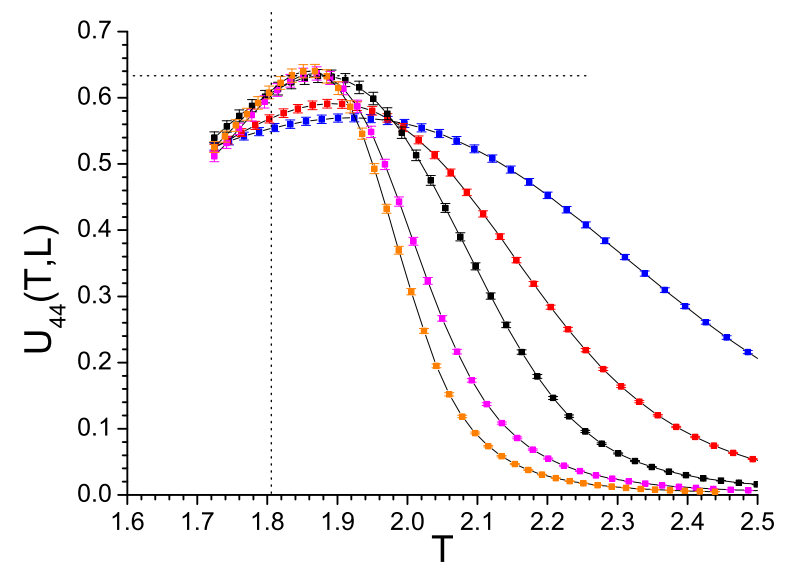

FIG. 15. Gaussian 4D ISG. Non-self-averaging parameter $U_{44}(T, L)$ against the temperature $T . L=4,6,8,10,12$ (blue, red, black, pink, orange) from right to left. The horizontal line is an extrapolation to criticality at $T=T_{c}$ (vertical line).

When scaled against the correlation length ratio, in the paramagnetic range $U_{22}(T, L)=\left[K_{5} \xi(T, L) / L\right]^{5}$ following the Aharony-Harris law [1], with $K_{5}=2.5(1)$. The peak locations correspond to $\xi(T, L) / L \approx 0.50$. As this ratio is greater than $[\xi / L]_{c}$ the locations of the $U_{n n}(T, L)$ peaks are at temperatures below $T_{c}$ and the peak temperatures move upwards towards $T_{c}$ with increasing $L$. The peak location extrapolated to infinite size provides an estimate for $T_{c}$, which is again limited by the statistical precision but which provides a useful independent check on the value of the ordering temperature. As the peak position changes are small, a quantitative scaling of the peak positions is not possible.

\section{DIMENSION 7 AND SUMMARY}

By this dimension, $N$ the number of spins per sample has become very large, $(N=823,543$ for $L=7)$, which imposes practical limits on the sizes and numbers of samples in the

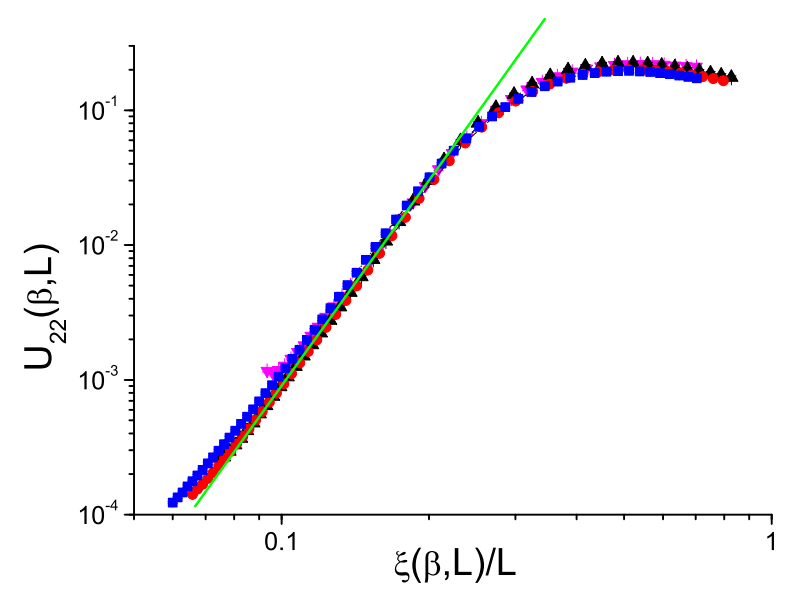

FIG. 16. Gaussian 5D ISG. Non-self-averaging parameter $U_{22}(T, L)$ against the normalized correlation length $\xi(T, L) / L . L=4$, $6,8,10$ (blue squares, red circles, black triangles, pink inverted triangles). The straight line has slope 5 .

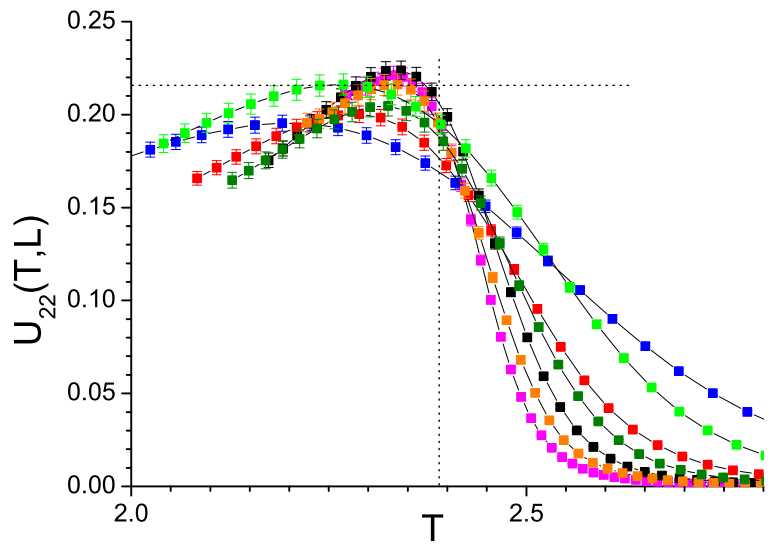

FIG. 17. Gaussian 5D ISG. Non-self-averaging parameter $U_{22}(T, L)$ against the temperature $T . L=4,5,6,7,8,9,10$ (blue, green, red, olive, black, orange, pink) from right to left on the right. The horizontal line is an extrapolation to criticality at $T=T_{c}$ (vertical line).

simulations. The simulations were carried out for $L=3$ to 7 with 512 samples at each $L$.

The dimension 7 bimodal ISG has an ordering temperature $T_{c}=3.39(1)$ estimated using the standard Binder cumulant crossing point technique [14] in agreement with the hightemperature series expansion (HTSE) estimates $T_{c}=3.37(2)$ [15] and $T_{c}=3.384(15)$ [16]. (Curiously the HTSE value given in Ref. [17] corresponds to $T_{c}=3.459$. We suspect a typographical error). As this dimension is above the upper critical dimension $d=6$, the critical exponents $\gamma=1$ and $v=1 / 2$ are known exactly. In this case in the paramagnetic regime $U_{22}(T, L)=\left[K_{7} \xi(T, L) / L\right]^{6}$, with an exponent which appears to be $\approx 6$ rather than 7 (Fig. 20). This could arise from the breakdown of the relations between scaling exponents above the upper critical dimension. Because of the limited number of samples and the small values of $L$ at this dimension, this estimate is not very precise.

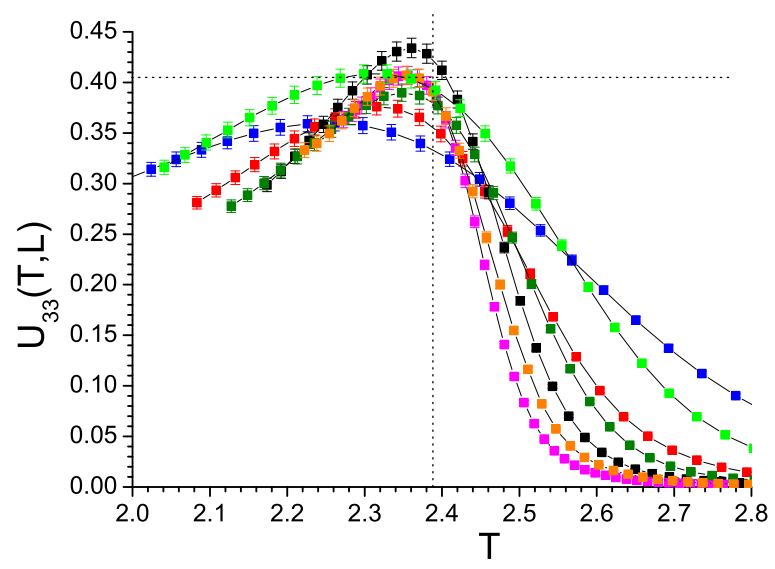

FIG. 18. Gaussian 5D ISG. Non-self-averaging parameter $U_{33}(T, L)$ against the temperature $T . L=4,5,6,7,8,9,10$ (blue, green, red, olive, black, orange, pink) from right to left on the right. The horizontal line is an extrapolation to criticality at $T=T_{c}$ (vertical line). 


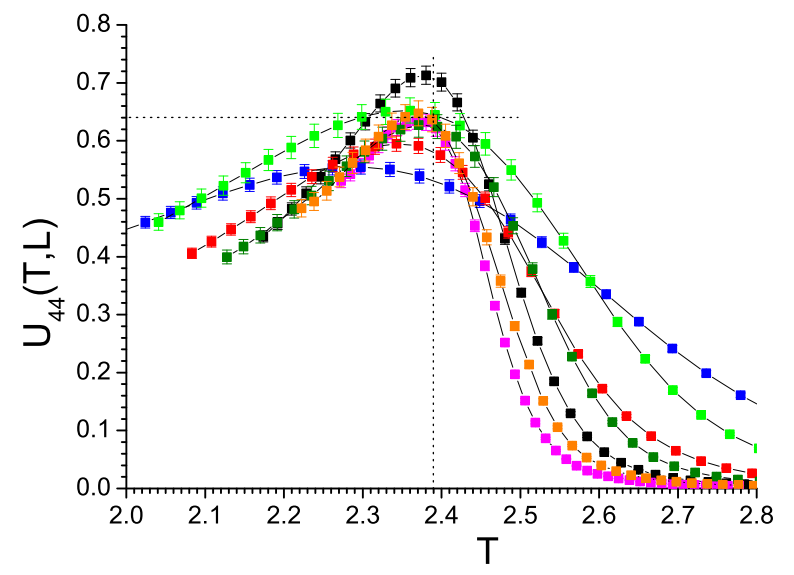

FIG. 19. Gaussian 5D ISG. Non-self-averaging parameter $U_{44}(T, L)$ against the temperature $T . L=4,5,6,7,8,9,10$ (blue, green, red, olive, black, orange, pink) from right to left on the right. The horizontal line is an extrapolation to criticality at $T=T_{c}$ (vertical line).

In the plot of $U_{22}(T, L)$ against $T$ (see Fig. 21), the $L$-independent critical finite-size crossing point value is $U_{22}\left(T_{c}\right) \approx 0.15$, and the $\left[U_{22}(T, L)\right]_{\max }$ peak heights are independent of $L$ and equal to $\approx 0.21$ to within the statistics, as for the other dimensions. The maxima locations move towards $T_{c}$ from within the ordered regime. This behavior is very similar to that observed in the mean field ISG SK model $[18,19]$, where again the $U_{22}(T, L)$ peak heights tend to about 0.21 .

The higher order $U_{33}(T, L)$ and $U_{44}(T, L)$ (Figs. 22 and 23) follow much the same pattern. Finally we have collected the ISG $U_{22}, U_{33}$, and $U_{44}$ peak height data for all the dimensions studied. These are displayed in such a way as to underline the interdimension regularities in Figs. 24, 25, and 26. Within the present statistics the peak heights are dimension independent for dimensions 3 to 7 and are slightly lower in dimension 2 .

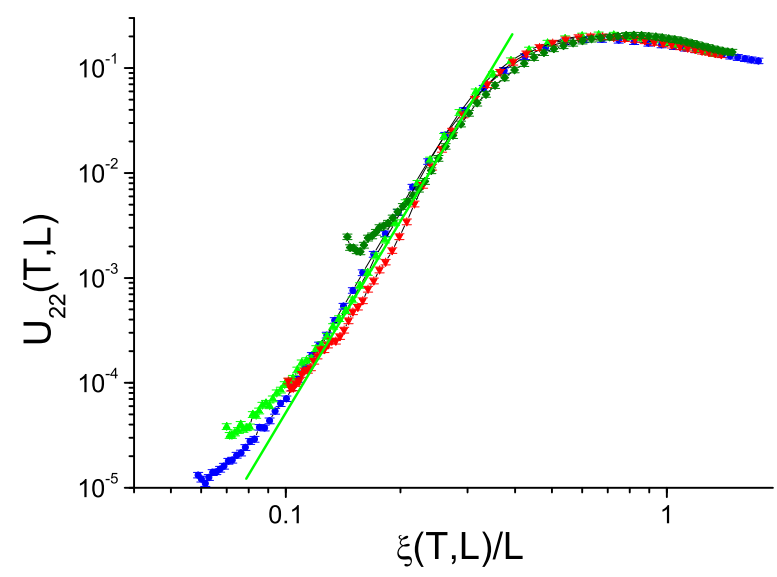

FIG. 20. Bimodal 7D ISG. Non-self-averaging parameter $U_{22}(T, L)$ against the normalized correlation length $\xi(T, L) / L . L=4$, 5, 6, 7 (blue circles, green triangles, red inverted triangles, olive diamonds). The straight line has slope 6 .

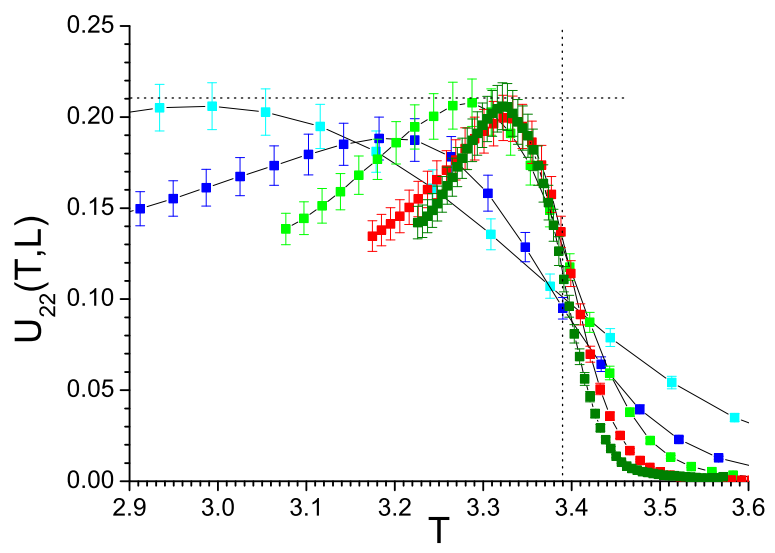

FIG. 21. Bimodal 7D ISG. Non-self-averaging parameter $U_{22}(T, L)$ against temperature $T . L=3,4,5,6,7$ (cyan, blue, green, red, olive) left to right on the left. Vertical line $T_{c}$. Horizontal line extrapolation.

\section{THE GAUGE GLASS}

The gauge glass (GG) is a canonical vector spin glass (see, for instance, Ref. [20]) where $X Y$ spins on a [hyper]cubic lattice of size $L$ interact through the Hamiltonian

$$
\mathcal{H}=-J \sum_{i j} \cos \left(\phi_{i}-\phi_{j}-A_{i j}\right),
$$

the sum ranging over near neighbors. The $\phi_{i}$ represent the angles of the spins, and the $A_{i j}$ are quenched random variables uniformly distributed between $[0,2 \pi] . J$ is conventionally set equal to 1 . Periodic boundary conditions are applied. The GG does not support chiral ordering. The GG in dimension 3 has a critical temperature $T_{c}=0.47(1)$ [20-22].

The non-self-averaging parameter $U_{22}(L, T)$ scales with $\xi(L, T) / L[21]$ and shows a maximum peak height independent of $L$ and a peak position $T_{\max }(L)$ near $\xi(L, T) / L=0.35$. The paramagnetic regime data [21] appear by inspection to be compatible with the Aharony-Harris rule $U_{22}(L, T) \sim$ $[\xi(L, T) / L]^{3}$ although the published data are not presented

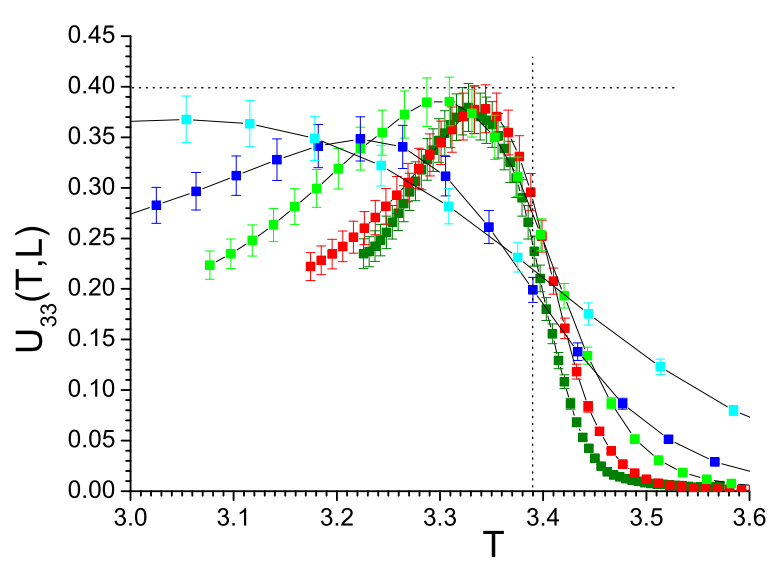

FIG. 22. Bimodal 7D ISG. Non-self-averaging parameter $U_{33}(T, L)$ against temperature $T . L=3,4,5,6,7$ (cyan, blue, green, red, olive) left to right on the left. Vertical line $T_{c}$. Horizontal line extrapolation. 


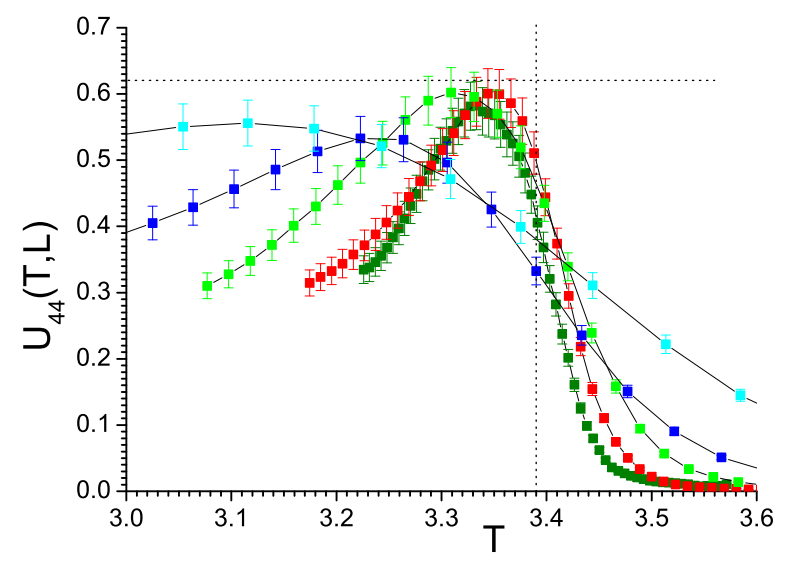

FIG. 23. Bimodal 7D ISG. Non-self-averaging parameter $U_{44}(T, L)$ against temperature $T . L=3,4,5,6,7$ (cyan, blue, green, red, olive) left to right on the left. Vertical line $T_{c}$. Horizontal line extrapolation.

in this way. As the critical correlation length ratio is $[\xi(L, T) / L]_{c}=0.54[21]$, the $U_{22}(L, T)$ peak temperature location moves downwards with $L$ and tends towards $T_{c}$. The 3D GG vector spin-glass $U_{22}(T, L)$ thus follows basically the same rules as followed by $U_{22}(T, L)$ in the ISG in 3D, except that the GG peak maximum is $\approx 0.10$ instead of 0.205 . Data on GGs in dimensions 2, 3, and 4 from measurements which were not designed to estimate the non-self-averaging parameter [22] are consistent with $U_{22}(T, L)$ peak values near 0.10 in each dimension. We can speculate that this family of spin-glass models also has its characteristic dimension-independent value of the non-self-averaging peak height.

\section{HEISENBERG AND $X Y$ SPIN GLASSES}

These spin glasses have the same Hamiltonian as the ISGs but with vector spins which are Heisenberg (three component) or $X Y$ (two component), respectively. Numerical measurements on Heisenberg spin glasses (HSGs) are of

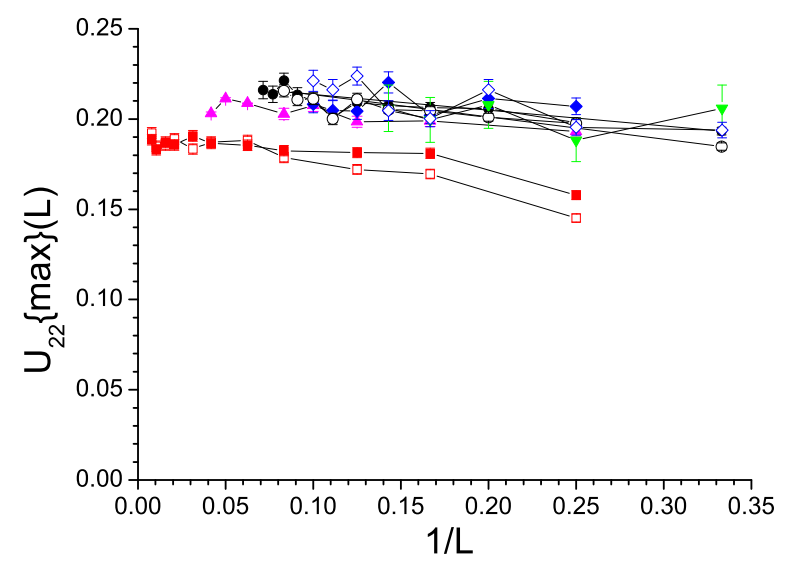

FIG. 24. Peak values of the non-self-averaging parameter $U_{22}(T, L)$ against inverse size $1 / L$ for all the ISGs studied. Open symbols are for Gaussian ISGs, closed for bimodal ISGs. Red squares $2 \mathrm{D}$, pink triangles $3 \mathrm{D}$, black circles $4 \mathrm{D}$, blue diamonds $5 \mathrm{D}$, green inverted triangles 7D.

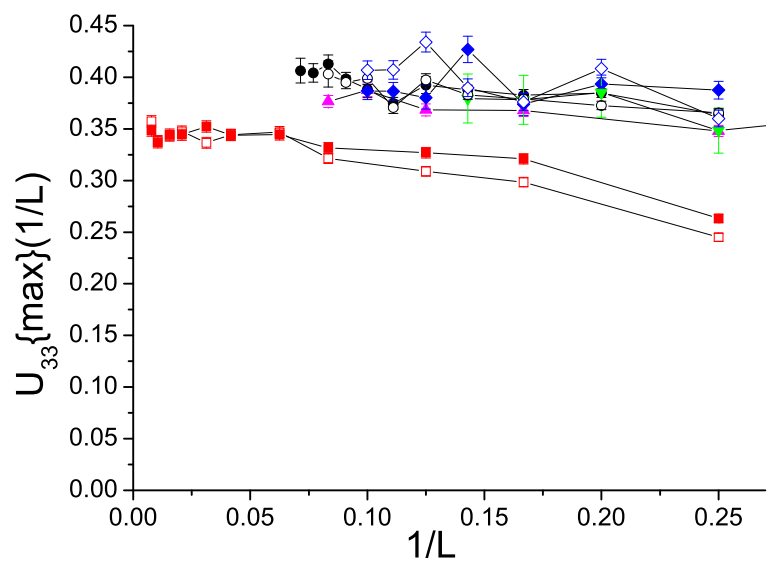

FIG. 25. Bimodal and Gaussian ISG non-self-averaging $U_{33}(L)$ peak values against inverse size $1 / L$. Closed symbols bimodal, open symbols Gaussian. Red squares 2D, pink triangles 3D, black circles $4 \mathrm{D}$, blue diamonds $5 \mathrm{D}$, green inverted triangles $7 \mathrm{D}$.

particular importance because the canonical experimental spin-glass dilute alloys (AuFe, $\mathbf{C u M n , ~ A g M n ) ~ a r e ~ a l l ~ H e i s e n - ~}$ berg systems, so it should be possible to understand the ordering mechanism in "real life" spin glasses on the basis of numerical data on Heisenberg models. We have no new data to report on these models, but it is of interest to consider published non-self-averaging data in the light of the ISG results.

Both Heisenberg and $X Y$ spin glasses can support chiralglass order as well as spin-glass order, and for many years there have been two conflicting interpretations of the numerical data on the ordering transitions in these models in dimension 3. According to the first interpretation, the ordering is spin-spin interaction driven; basically the ordering process is much the same as in ISGs, and the chiral order follows on as a geometrically necessary consequence of the onset of spin order, without the chiral interactions playing any significant role in the spin glass transition [23-27]. The alternative

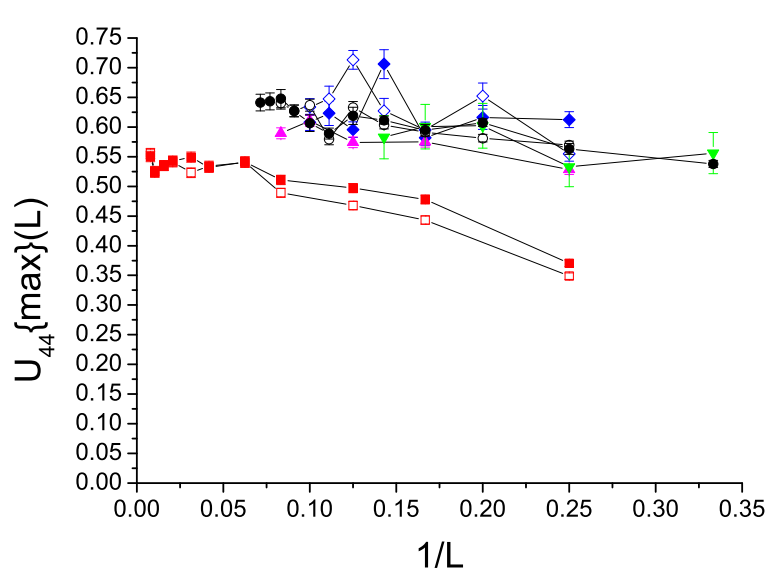

FIG. 26. Bimodal and Gaussian ISG non-self-averaging $U_{44}(L)$ peak values against inverse size $1 / L$. Closed symbols bimodal, open symbols Gaussian. Red squares 2D, pink triangles 3D, black circles $4 \mathrm{D}$, blue diamonds $5 \mathrm{D}$, green inverted triangles $7 \mathrm{D}$. 
interpretation is that the driving role in 3D HSG or $X Y \mathrm{SG}$ ordering is played by the chirality, so that there is first a chiral order onset followed at a lower temperature by spin ordering transition [28-31]. (Similar disagreements concerning fully frustrated 2D $X Y$ models were resolved definitively in favor of a distinct chiral-order transition just above a spin-order transition [32,33].) The arguments of both schools to support their respective interpretations in the $3 \mathrm{D}$ HSG and $X Y \mathrm{SG}$ models have been essentially based on analyses of the data for the crossing points of the dimensionless normalized spin and chiral $\left(s\right.$ and $c$ ) correlation lengths $\xi_{s}(T, L) / L$ and $\xi_{c}(T, L) / L$.

The numerical simulations in the spin glasses are even more demanding than in the fully frustrated models, and because of intrinsic finite-size corrections and the need to reach strict equilibration at each $L$, extrapolations to infinite $L$ in order to estimate the ThL crossing point locations are delicate. As simulations were extended to larger sizes in successive Gaussian HSG and XYSG measurements interpreted on the spin-driven ordering scenario, the joint spin or chiral crossover temperature was estimated to be $T_{c}(H S G) \approx 0.160$ [23], $T_{c}(H S G) \approx 0.145$ with a KTB-like critical line [24], marginal but very similar spin and chiral behavior (XYSG and HSG) [25,26], and most recently $T_{c}(H S G) \approx 0.120$ [27]. No non-self-averaging results were reported. From detailed 3D bimodal and Gaussian HSG and 3D Gaussian XYSG measurements, the two separate transition temperatures on the chiral-driven ordering scenario are estimated to be (bimodal HSG) [29], $T_{c}(c)=0.194(5)$ and $T_{c}(s) \leqslant 0.15$, (Gaussian HSG) $T_{c}(c)=0.143(3)$ and $T_{c}(s)=0.125(+0.006 /-$ 0.012 ) [30], and (XYSG) $T_{c}(c)=0.308(5)$ and $T_{c}(s)=$ $0.274(3)$ [31]. Non-self-averaging data were shown in each case.

In the light of the ISG results reported above, it would appear that in Heisenberg and $X Y$ spin glasses non-selfaveraging could provide an independent primary numerical criterion for spin and/or chiral ordering much less sensitive to finite-size effects and to strict equilibration (as already suggested in Ref. [29]). On the first (spin-driven ordering) scenario one would expect the spin non-self-averaging parameter $U_{22 s}(T, L)$ to follow much the same rules as for the ISG or the GG chiral-free vector spin-glass cases discussed above, with a peak location moving towards an upper spin-ordering temperature $T_{c}(s)$ as $L$ increases, and a regular behavior reflecting $U_{22 s}(T, L) \sim\left[\xi_{s}(T, L) / L\right]^{3}$ in the paramagnetic regime above $T_{c}(s)$. On this interpretation the chiral $U_{22 c}(T, L)$ would be weaker than the $U_{22 s}(T, L)$; if a $U_{22 c}(T, L)$ peak exists, it would be located at a temperature below or possibly at the ThL $U_{22 s}(T, L)$ peak.

On the second (chiral-driven order) scenario, it would be the chiral $U_{22 c}(T, L)$ which would show a peak first, with a peak location tending towards the (upper) chiral ordering temperature $T_{c}(c)$ as $L$ increases. In the paramagnetic regime one would expect a regular behavior of the chiral non-self-ordering $U_{22 c}(T, L)$ with increasing $L$, governed by $U_{22 c}(T, L) \sim\left[\xi_{c}(T, L) / L\right]^{3}$. On this scenario the spin $U_{22 s}(T, L)$ would then show a peak location somewhere below the chiral $U_{22 c}(T, L)$ peak, with a location tending towards an ordering temperature $T_{c}(s)$ below $T_{c}(c)$, together with a paramagnetic regime $U_{22 s}(T, L)$ behavior behaving irregularly at least at small $L$ because the paramagnetic spin ordering is perturbed by the dominant onset of chiral order.

Very informative non-self-averaging data have been published on the 3D HSG with bimodal interactions [29], on the 3D HSG with Gaussian interactions [30], and on the 3D Gaussian $X Y$ SG [31]. In each case the pattern is the same. First, there is a strong $U_{22 c}(T, L)$ peak at an almost $L$-independent temperature $T \approx 0.19, T \approx 0.145, T \approx 0.31$, respectively, so in each case close to the $T_{c}(c)$ value estimated independently from other criteria [29-31]. In the paramagnetic regime there is a regular narrowing in temperature of the $U_{22 c}(T, L)$ peak with increasing $L$ which appears compatible with the AharonyHarris law $U_{22 c}(T, L) \sim\left[\xi_{c}(T, L) / L\right]^{3}$ though the data are not presented in this form. Second, in each case, the spin $U_{22 s}(T, L)$ peak is either not visible (HSGs) or is marginal ( $X Y \mathrm{SG}$ ) down to the lowest temperature at which non-selfaveraging measurements were made, $T \approx 0.145, T \approx 0.11$ to 0.13 depending on $L$, and $T \approx 0.24$ to 0.275 depending on $L$ in the three cases.

Thus the non-self-averaging data $U_{22 c}(T, L)$ and $U_{22 s}(T, L)$ in the three models [29-31] appear by inspection to be fully compatible with the chiral-driven ordering interpretation [28-31], but incompatible with the HSG behavior expected on the spin-driven ordering scenarios [23-27], unless there are strong finite-size effects such that a $U_{22 s}(T, L)$ peak (invisible in the present data) moves up in temperature with increasing $L$ to rejoin the $U_{22 c}(T, L)$ peak. Independent arguments in favor of the chiral-driven ordering are given in [34].

It would appear that further numerical measurements designed specifically to study the non-self-averaging could resolve conclusively the question of the driving mechanism for ordering in Heisenberg and $X Y$ spin glasses.

\section{CONCLUSION}

The non-self-averaging data on ISGs in all dimensions show a remarkable regularity. In each dimension there is a peak as a function of temperature in the standard nonself-averaging parameter $U_{22}(T, L)$ and in the higher-order parameters $U_{33}(T, L)$ and $U_{44}(T, L)$ whose values are $L$ independent after weak small size effects. As can be seen in Figs. 24, 25, and 26 the peak values $U_{22}(T, L)_{\max } \approx 0.21$, $U_{33}(T, L)_{\max } \approx 0.40, U_{44}(T, L)_{\max } \approx 0.62$ are independent of dimension to within the statistics for dimensions $3,4,5$, and 7 (and for infinite dimension SK model [18,19] $U_{22}$ measurements), with a slightly smaller value in dimension 2 . In the paramagnetic regime above the peak the Aharony-Harris renormalization group law [1] $U_{n n}(T, L)=\left(K_{d} \xi(T, L) / L\right)^{d}$ is obeyed, with $K_{d}\left(U_{22}\right) \approx 2.6$ for all dimensions. Both of these empirical observations can be classed tentatively as "hyperuniversal behavior."

Published [21] and unpublished [22] data on the GG, a vector spin glass which does not support chirality, suggest that non-self-averaging rules analogous to those that hold in the ISGs appear to apply but with a different characteristic peak height $U_{22}(T, L)_{\max } \approx 0.10$.

$X Y$ and Heisenberg spin glasses can support chiral ordering as well as spin ordering. In the light of the non-self-averaging behavior reported above for the ISG models, the published spin and chiral non-self-averaging data [29-31] in 3D Heisenberg 
and $X Y$ models appear to be incompatible with a spin-driven ordering scenario [23-27] but support the alternative conclusion that the spin-glass ordering in these models is chiraldriven rather than spin-driven, in the Kawamura scenario [28]. An important implication would be that order in the canonical experimental Heisenberg spin glasses is also chirality driven. We suggest that simulations directly designed to study the non-self-averaging in these systems should be undertaken.

\section{ACKNOWLEDGMENTS}

We would like to thank H. Kawamura for helpful comments. The computations were performed on resources provided by the Swedish National Infrastructure for Computing (SNIC) at the High Performance Computing Center North (HPC2N) and Chalmers Centre for Computational Science and Engineering (C3SE).
[1] A. Aharony and A. B. Harris, Phys. Rev. Lett. 77, 3700 (1996)

[2] H. G. Katzgraber, M. Körner, and A. P. Young, Phys. Rev. B 73, 224432 (2006).

[3] P. H. Lundow and I. A. Campbell, Phys. Rev. E 91, 042121 (2015); Physica A 434, 181 (2015).

[4] P. H. Lundow and I. A. Campbell, arXiv:1506.07141.

[5] A. K. Hartmann and A. P. Young, Phys. Rev. B 64, 180404(R) (2001).

[6] M. Ohzeki and H. Nishimori, J. Phys. A: Math. Theor. 42, 332001 (2009).

[7] T. Jörg, J. Lukic, E. Marinari, and O. C. Martin, Phys. Rev. Lett. 96, 237205 (2006).

[8] F. Parisen Toldin, A. Pelissetto, and E. Vicari, Phys. Rev. E 82, 021106 (2010); 84, 051116 (2011).

[9] P. E. Theodorakis, I. Georgiou, and N. G. Fytas, Phys. Rev. E 87, 032119 (2013).

[10] M. Hasenbusch, A. Pelissetto, and E. Vicari, Phys. Rev. B 78, 214205 (2008), and EPAPS Document No. E-PRBMDO-78003845.

[11] M. Baity-Jesy et al., Phys. Rev. B 88, 224416 (2013).

[12] M. Palassini, M. Sales, and F. Ritort, Phys. Rev. B 68, 224430 (2003).

[13] T. Jörg and H. G. Katzgraber, Phys. Rev. Lett. 101, 197205 (2008).

[14] P. H. Lundow and I. A. Campbell (unpublished).

[15] R. R. P. Singh and S. Chakravarty, Phys. Rev. Lett. 57, 245 (1986).

[16] D. Daboul, I. Chang, and A. Aharony, Eur. Phys. J. B 41, 231 (2004).
[17] L. Klein, J. Adler, A. Aharony, A. B. Harris, and Y. Meir, Phys. Rev. B 43, 11249 (1991).

[18] K. Hukushima and H. Kawamura, Phys. Rev. E 62, 3360 (2000).

[19] M. Picco, F. Ritort, and M. Sales, Eur. Phys. J. B 19, 565 (2001).

[20] T. Olson and A. P. Young, Phys. Rev. B 61, 12467 (2000).

[21] V. Alba and E. Vicari, Phys. Rev. B 83, 094203 (2011).

[22] H. G. Katzgraber and I. A. Campbell, Phys. Rev. B 69, 094413 (2004).

[23] L. W. Lee and A. P. Young, Phys. Rev. Lett. 90, 227203 (2003).

[24] I. Campos, M. Cotallo-Aban, V. Martín-Mayor, S. Perez-Gaviro, and A. Tarancon, Phys. Rev. Lett. 97, 217204 (2006).

[25] L. W. Lee and A. P. Young, Phys. Rev. B 76, 024405 (2007).

[26] J. H. Pixley and A. P. Young, Phys. Rev. B 78, 014419 (2008).

[27] L. A. Fernandez, V. Martín-Mayor, S. Perez-Gaviro, A. Tarancon, and A. P. Young, Phys. Rev. B 80, 024422 (2009).

[28] H. Kawamura, J. Phys. Soc. Jpn. 79, 011007 (2010).

[29] K. Hukushima and H. Kawamura, Phys. Rev. B 72, 144416 (2005)

[30] D. X. Viet and H. Kawamura, Phys. Rev. Lett. 102, 027202 (2009); Phys. Rev. B 80, 064418 (2009).

[31] T. Obuchi and H. Kawamura, Phys. Rev. B 87, 174438 (2013).

[32] M. Hasenbusch, A. Pelissetto, and E. Vicari, Phys. Rev. B 72, 184502 (2005).

[33] S. Okumura, H. Yoshino, and H. Kawamura, Phys. Rev. B 83, 094429 (2011).

[34] I. A. Campbell and C. M. C. Petit, J. Phys. Soc. Japan 79, 011006 (2010). 